\title{
Obsessive Compulsive Inventory - Child Version (OCI-CV): Confirmatory factor analysis, reliability, validity and correlates among Nigerian adolescents
}

\author{
Tolulope Opakunle ${ }^{1}$, Olutayo Aloba², Adesanmi Akinsulore ${ }^{2}$ \\ 1. State Specialist Hospital, Osogbo, Osun State, Nigeria \\ 2. Obafemi Awolowo University Teaching Hospitals Complex, Ile-Ife, Osun State, Nigeria
}

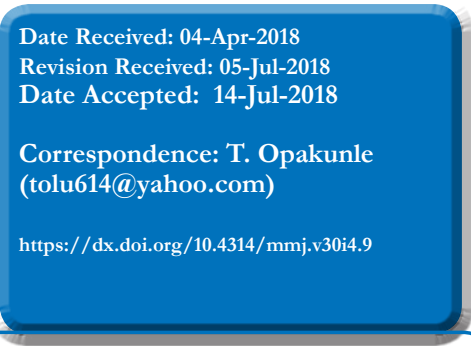

\section{Background}

\section{Abstract}

Obsessive compulsive symptoms (OCS) have not been studied among Nigerian adolescents, despite studies in developed countries reporting that almost $90 \%$ of affected adolescents do not receive any treatment. The availability of a psychometrically valid and reliable instrument will serve as an initial step towards the diagnosis and subsequent treatment of Nigerian adolescent obsessive compulsive disorder $(\mathrm{OCD})$.

Aims

The aim of this study was to examine the psychometric characteristics as well as correlates of the Obsessive Compulsive Inventory Child Version (OCI-CV) in a non-clinical sample of Nigerian adolescents.

Methods

This was a cross-sectional study involving 1017 adolescents who completed the Obsessive Compulsive Inventory-Child Version (OCICV), the Hospital Anxiety and Depression Scale (HADS), General Health Questionnaire-12 (GHQ-12) and Rosenberg's Self-Esteem Scale (RSES). The model fit of the OCI-CV 6 factors was examined through confirmatory factor analysis. Reliability was determined by calculating the McDonald's Omega hierarchical ( $\omega \mathrm{h}$ ) values while its concurrent validity was examined through correlational analyses. Results

The overall internal consistency of the OCI-CV was 0.96 . The indices of fitness obtained on subjecting the 21 items of the OCI-CV to CFA indicated modestly acceptable indices of fitness despite satisfactory item loading on the 6 subscales. Statistically significant correlations were observed between OCI-CV and the other study measures. OCS was reported by $37.8 \%$.

Conclusion

The OCI-CV has exhibited satisfactory psychometric properties among Nigerian adolescents.

Key words - Obsessive Compulsive Inventory - Child Version, Nigerian, adolescents, factor analysis, reliability, validity

\section{Introduction}

Obsessive compulsive disorder (OCD) is a chronic and potentially disabling psychiatric disorder with a lifetime prevalence of $2 \%$ to $3 \%$ among adults ${ }^{1}$. It often begins in childhood or early adolescence ${ }^{2}$. However, it has been reported that paediatric OCD is frequently under-diagnosed and almost $90 \%$ of affected children and adolescents do not receive any form of pharmacological or psychological treatment, especially those with mild and moderate symptom severity $^{3}$. A number of factors have been identified as contributing to the delay in the identification and subsequent treatment of OCD among adolescents. These include the secretive nature of the disorder, the lack of insight in children and the high rates of associated co-morbidities among adolescents ${ }^{4,5}$. The prevalence of obsessive compulsive symptoms (OCS) among adolescents varies across different countries. It ranges from $2.7 \%$ to $19.4 \%$ in the developed western countries ${ }^{6-11}$. The few studies in African countries reported prevalence rates ranging from $13.3 \%$ to $43.1 \%{ }^{12-14}$. However, in spite of these various studies, OCS in children and adolescents are still underestimated and understudied ${ }^{2}$. OCS can persist over several years ${ }^{15}$. Their presence among adolescents even in mild severity is associated with an increased risk of co-occurring psychiatric disorders and varying degrees of functional and academic impairments ${ }^{8,9}$. Obsessive compulsive symptoms are associated with higher frequencies of co-morbid tic disorders, disruptive behavioural disorders and attention deficit hyperactivity disorder ${ }^{16}$. They are also associated with suicidal ideation and depressive symptoms ${ }^{17,18}$. Studies have reported a relationship between depression and $\mathrm{OCS}^{8,19}$. It is believed that depressive symptoms could be due to the distress caused by the impairment of OCS in children ${ }^{20}$. A study also reported reduced levels of self-esteem in patients with OCS when compared to those without OCS ${ }^{21}$. Furthermore, there is a relationship between obsessive compulsive disorder and other anxiety disorders ${ }^{22}$. A study reported a worse prognosis in those with OCS who have a family history of other anxiety disorders ${ }^{15}$. Therefore, it is recommended that adolescents be routinely screened for the presence of OCS for early detection and subsequent treatment ${ }^{3}$.

A significant number of psychometrically validated scales are available for the screening of OCS among children and adolescents. Among these measures are the Children's Yale Brown Obsessive Compulsive Symptoms (CY-BOCS) ${ }^{23}$, Children's Florida Obsessive Compulsive Inventory 
$(\mathrm{CFOCI})^{24}$, Leyton Obsessive Inventory - Children Version (LOI-CV) ${ }^{25}$ and Children's Obsessive Compulsive Inventory $(\mathrm{COCI})^{26}$. The $\mathrm{CFOCI}$ demonstrated good internal consistency (0.89) and significantly correlated with Yale Brown Obsessive Compulsive Symptoms. However, it doesn't assess the severity of individual symptoms ${ }^{24}$. The LOI-CV also had satisfactory validity but its scores did not correlate significantly with other clinical measures of obsessive behaviours ${ }^{25}$.

The CY-BOCS is the most commonly used scale to assess OCS in children and adolescents, and also to monitor treatment outcome ${ }^{23}$. Its internal consistency was satisfactory (Cronbach's alpha -0.87$)^{23}$. The CY-BOCS checklist is currently divided into 19-symptom categories that measure each item's severity of OCS. This structure, however, has been challenged by studies that have examined its factorial structure $^{27}$. It is a clinician-administered measure that requires training In addition, it is expensive and its usage is time-consuming ${ }^{26}$.

The Obsessive Compulsive Inventory - Child Version (OCI-CV) was developed by Foa and colleagues in $2010^{26}$. It is a 21 -item subjectively completed instrument for the quantitative assessment of OCS in children and adolescents aged from 7 to 17 years. The OCI-CV demonstrated excellent psychometric properties in a clinical sample of 109 children and adolescents diagnosed with $\mathrm{OCD}^{26}$. The exploratory factor analysis of this version yielded six factors which were labeled; Doubt/Checking, Obsessions, Hoarding, Washing, Ordering, and Neutralizing. The OCI-CV items showed good internal consistency with Cronbach's alpha value greater than 0.81 for the whole scale and for the subscales ${ }^{26}$. The test-retest reliability coefficients values were 0.77 for the entire scale, and a range of 0.68 to 0.89 for the 6 subscales. The correlations between OCI-CV and the other measures of paediatric OCD, such as CY-BOCS were statistically significant $^{28}$.

The OCI-CV is easily completed and has exhibited excellent psychometric properties across different cultures ${ }^{26,28-33}$. In a Spanish population, a six-factor model was observed and internal consistency (Cronbach's alpha) for the total score was satisfactory at $0.83^{28}$. In an Italian population of children and adolescents, a model consisting of six factors exhibited satisfactory indices of fitness (Bentler-Bonett Normed Fit Index (NFI) - 0.96, Tucker Lewis Index (TLI) - 0.96, Comparative Fit Index $(\mathrm{CFI})-0.97)^{33}$. Generally, the reliability of the OCI-CV as well as its subscales has been described as satisfactory, ranging from good to acceptable ${ }^{33}$. A study in the United States of America also reported a sixfactor model with a good internal consistency ${ }^{29}$. The internal consistencies for the OCI-CV subscales ranged from poor to good and the Inventory correlated modestly with the CYBOCS severity scale ${ }^{29}$.

It has been suggested that there is a need for the examination of the psychometric properties of the OCI-CV among diverse children and adolescent populations in terms of different races and cultures ${ }^{28}$. This is to avoid undue generalizations, in addition to facilitating the dissemination of evidence based assessment procedures ${ }^{28}$. In Nigeria, there is lack of research regarding the screening, prevalence, and correlates of OCS among the adolescent population. The availability of a psychometrically valid and reliable OCS measure will serve as an initial step at identifying OCS among the population of Nigerian adolescents. We decided on OCI-
$\mathrm{CV}$ due to a number of reasons. It has been widely used in different cultures and populations. It is a relatively brief selfcompleted measure that was developed about 7 years ago and made freely available for online downloading and utilisation after obtaining approval from the Inventory's developers. An extensive electronic literature search revealed that the psychometric characteristics of the OCI-CV have not been described among the children and adolescent populations in Nigeria. Therefore, the aims of this study were to: (a) examine the factor structure of the OCI-CV in a non-clinical sample of Nigerian adolescents, (b) examine the reliability of the OCI-CV and its subscales, (c) examine the validity of the OCI-CV in the same sample, and; (d) determine, via multiple linear regression analyses, the variables that would significantly predict the OCI-CV subscales score among our respondents.

\section{Methods}

\section{Study setting and design}

This cross-sectional study was conducted in Osogbo, the capital city of Osun State in south-western Nigeria. The occupations of most inhabitants of the ancient city include public/civil service, farming, trading and artisanship. The study population consists of adolescents in all the public high schools in Osogbo. There are a total of 4 government high schools in the city. The students are from different ethnic groups. The medium of expression and teaching is English language in all the schools in south-western Nigeria.

We adopted a multistage stratified sampling technique. In the first stage, 3 classes were randomly chosen from each of the 3 levels of senior secondary classes in each of the high schools. This made a total of 9 classes per school and 36 classes in total. The second stage involved the selection of 30 students per class by balloting. This yielded a total sample of 1080 adolescents which represented about $10 \%$ of the total population of the high school students with age range of 13 to 19 years.

\section{Procedure}

The approval for the study protocol was obtained from the Research and Ethics Committee of the Ladoke Akintola University of Technology Teaching Hospital (LTH), Osogbo, Osun State. Permission was also obtained from the State Ministry of Education, and from the heads of the selected schools. The inclusion criteria included adolescent students within the age range of 13 to 19 years, those who gave assent to participate in the study and those with parental consent for the study participation. The students were given a form to give to their parents explaining the study in terms of its nature and purpose. The parents gave or refused consent by indicating it on the form.

\section{Instruments}

The sociodemographic questionnaire variables included age, gender (male and female), religion (Christianity, Islam, and Indigenous traditional) and the family background (Monogamous, Polygamous and Single parent). The OCI$\mathrm{CV}$ is a 21-item self-report measure that assesses obsessive compulsive symptoms in children and adolescents aged 7 to 17 years over the preceding one month ${ }^{26}$. It is a Likert-type rating scale, scored 0 to 2 (where $0=$ never, $1=$ sometimes and 2 = always). The highest possible score is 42 . Higher score on the OCI-CV means greater severity of symptoms. The OCI-CV is based on the 42-item OCI which has been 
utilized in a clinical sample of adult patients in Nigeria ${ }^{34}$.

The Hospital Anxiety and Depression Scale (HADS) is a 14item scale with 2 subscales; the anxiety and the depression subscales. Each scale is composed of 7 items $^{35}$. Each item is scored on a 4 point Likert scale (0-3). The total score on each subscale ranges from 0 to 21 . Higher scores on the subscales reflect higher anxiety and depressive symptoms. Satisfactory reliability and validity of the HADS as a screening instrument for anxiety and depressive disorders have been described within samples of the non-clinical and clinical populations in Nigeria ${ }^{36}$. In this study, the internal consistencies of the HADS-Anxiety (McDonald's omega hierarchical ( $\omega$ h) - 0.85) and Depression ( $\omega$ h - 0.73) subscales were satisfactory.

The General Health Questionnaire - 12 (GHQ-12) is a 12 -item self-rated questionnaire that is used in the quick assessment of general psychopathology ${ }^{37}$. The questionnaire has been validated in Nigeria ${ }^{38}$. We adopted the $0-0-1-$ 1 scoring approach ${ }^{38}$. Total score on the questionnaire ranges from 0 to 12 , with higher scores reflecting greater psychological distress and the likelihood of screening positive for a psychiatric disorder ${ }^{39}$. A cut-off score of 3 and above is indicative of probable psychiatric morbidity. The reliability of the GHQ-12 in this study was modestly satisfactory ( $\omega \mathrm{h}$ - 0.64). The Rosenberg Self-Esteem Scale (RSES) is a 10item questionnaire with items scored on a four-point Likert scale from "strongly agree" to "strongly disagree". Five of the scale's items are reverse-scored. Higher scores on the scale reflect higher levels of self-esteem ${ }^{40}$. Satisfactory reliability and validity have been described among Nigerian adolescents $^{41}$. The items of the RSES in this study had satisfactory internal consistency ( $\omega$ h - 0.87).

\section{Statistical analysis}

Statistical analyses were performed with the Statistical Product and Service Solutions (SPSS) 21st version and R Psych Package version 3.4.2. Descriptive statistics such as the mean (standard deviation) and frequency (percentages) were utilised in depicting the adolescents' sociodemographic variables and scores on the OCI-CV and the other study measures. Applying the SPSS $20^{\text {th }}$ version of the Analysis of Moment Structures (AMOS) software, we conducted a confirmatory factor analysis (CFA) on the total sample based on the previously established 6-factor structure reported by both the original authors of OCI-CV and the other studies in western countries ${ }^{26,28,30,33}$. A number of indices of fitness which included the Comparative Fit Index (CFI), Incremental Fit Index (IFI), Normed Fit Index (NFI), Standardized Root Mean Square Residual (SRMR), and the Root Mean Square Error of Approximation (RMSEA) were examined. Acceptable indices of fitness for the CFA model were based on the revised criteria according to Kline (2015), in which the CFI, IFI and NFI values will be $\geq 0.90$; a SRMR value of less than 0.80 and a RMSEA value of less than $0.80^{42}$.

The concurrent validity of the OCI-CV was examined through correlational analyses with the HADS Anxiety and Depressive subscales, the RSES and GHQ-12 mean scores. The reliabilities of the OCI-CV, both globally and in terms of its 6 subscales, were determined by calculating their McDonald's omega hierarchical (wh) values. Finally, linear regression analyses were performed to determine the socio-demographic and study measure variables that significantly predicted the OCI-CV subscale score among the respondents.

\section{Results}

\section{Socio-demographic and study measure characteristics of the respondents $(n=1017)$}

A total of 1080 secondary school students were contacted, out of which 36 declined to participate in the study and 27 provided incomplete data. Hence, only 1017 were available for analysis. Table 1 shows that the mean age of the students was 14.83 years $(\mathrm{SD}=1.38)$. Females constituted $50.9 \%$ of the sample. The mean total scores on the Anxiety and Depression subscales of the HADS were 5.67 (SD = 4.09) and $2.47(\mathrm{SD}=3.73)$ respectively. The mean score on the OCI-CV and its 6 subscales are also shown. The presence of OCS was observed in $37.8 \%$ of the respondents.

Table 1: Sociodemographic and study measure characteristics of the respondents $(n=1017)$

\begin{tabular}{|c|c|}
\hline Variable & $\begin{array}{l}\text { Mean (SD) } \\
\text { Frequency }(\%) /[\text { Range] }\end{array}$ \\
\hline \multicolumn{2}{|l|}{ Gender: } \\
\hline Male & $499(49.1 \%)$ \\
\hline Female & $518(50.9 \%)$ \\
\hline \multicolumn{2}{|l|}{ Family background: } \\
\hline Monogamous & $661(65.0 \%)$ \\
\hline Polygamous & $278(27.3 \%)$ \\
\hline Single parent & $78(7.7 \%)$ \\
\hline Age & $14.83(1.38)[13-19]$ \\
\hline $\mathrm{OCl}-\mathrm{CV}$ total score & $5.77(8.92)[0-37]$ \\
\hline Doubting/checking & $1.33(2.20)[0-10]$ \\
\hline Obsession & $1.08(1.81)[0-8]$ \\
\hline Hoarding & $0.76(1.30)[0-6]$ \\
\hline Washing & $1.04(1.66)[0-6]$ \\
\hline Ordering & $0.92(1.66)[0-6]$ \\
\hline Neutralizing & $0.64(1.20)[0-6]$ \\
\hline
\end{tabular}

HADS-Anxiety $\quad 5.67(4.09)[1-18]$

HADS-Depression $\quad 2.47(3.73)[0-17]$

Self-Esteem $21.73(3.63)[5-26]$

GHQ-12 $\quad 0.83(1.85)[0-12]$

OCS:

$\begin{array}{ll}\text { Present } & 384(37.8 \%) \\ \text { Absent } & 633(62.2 \%)\end{array}$

\section{Descriptive characteristics and reliability of the OCI-CV among the respondents $(n=1017)$}

As demonstrated in table 2 below, it can be observed that the corrected item-total correlations of the 21 items ranged from 0.55 to 0.80 . It can also be observed that the deletion of any of the Inventory's items did not significantly increase its overall reliability. The internal consistency for the overall OCI-CV was excellent ( $\omega \mathrm{h}-0.96)$. There was no evidence of multicollinearity among the 21 items of the OCI-CV. 
Table 2: Descriptive characteristics of the 21 items of the OCI-CV and overall reliability $(n=1017)$

\begin{tabular}{|c|c|c|c|}
\hline \multirow[t]{3}{*}{ Item / Subscale name } & Mean (SD) & Corrected & McDonald's \\
\hline & & item-total & Omega if item \\
\hline & & correlation & is deleted \\
\hline
\end{tabular}

Doubting / Checking

4. check many things over $0.40(0.70)$

0.78

0.95

and over.

0.68

0.96

sure if I really did them.

13. still worry that I didn't $0.21(0.47)$ finish things.

0.74

0.95

15. check doors, windows, $0.24(0.56)$ and drawers over and over

20 . when I do something very $0.25(0.54)$

carefully I don't think I did.

\section{Obsession}

1. think about bad things and $0.26(0.51)$ can't stop.

11. upset by bad thoughts. $\quad 0.25(0.58)$

14. get upset by bad thoughts $0.26(0.57)$

that pop into my head

18. bad thought comes into $0.30(0.62)$

my head, I say certain things.

0.68

0.67

0.96

0.96

Hoarding

$0.24(0.53)$

0.58

0.96

7. collect things I don't really $0.20(0.47)$ need.

16. don't throw things away $0.32(0.64)$

0.80

0.95

Washing

2. feel like I must wash and $0.44(0.76)$ clean.

10. worry about things being $0.22(0.62)$ clean.

21. wash my hands more.

$0.38(0.75)$

Ordering

8. get upset if my stuff is not in $0.29(0.76)$

0.76

0.95

the right order.

17. get upset if people change $0.30(0.80)$

the way I arrange things.

19. need things to be in a way. $0.33(0.79)$

0.80

0.79

0.95

\section{Neutralizing}

6 . need to count.

$0.25(0.69)$

0.69

0.96

9. get behind in my school- $0.20(0.55)$

work because I repeat things

12. say some numbers over $0.19(0.64)$

0.55

0.96

0.64

0.96

and over

Colinearity statistics revealed tolerance values close to 1.0 for all the items. In addition, the Condition Indexes for all the items were lower than $30^{43}$. Therefore, we had no reason to eliminate any of the Inventory's items. In terms of the subscales' items internal consistencies, the Doubting/ Checking, Obsession, Hoarding, Washing, Ordering and Neutralizing subscales had $\omega$ h values of $0.88,0.83,0.71,0.80$,
0.86 and 0.69 respectively. The indices of fitness obtained on subjecting the 21 items of the OCI-CV to CFA and after some residual error co-variations indicated modestly acceptable indices of fitness ( $\mathrm{IFI}=0.917$; CFI $=0.916$; $\mathrm{NFI}=0.906 ;$ SRMR $=0.041 ; \mathrm{RMSEA}=0.086 / 90 \% \mathrm{CI}=$ $0.082-0.096)$. The CFA path analysis indicating the items loading and standardized estimated for the 6 subscales of the OCI-CV are depicted in figure 1. Item loadings on the OCICV subscales ranged from 0.68 to 0.79 for the Doubting/ Checking subscale, 0.58 to 0.76 for the Obsession subscale, 0.57 to 0.80 for the Hoarding subscale, 0.65 to 0.81 for the Washing subscale, 0.77 to 0.85 for the Ordering subscale and 0.56 to 0.67 for the Neutralizing subscale.



DC - Doubting/Checking WASH - Washing OCS - Obsessive Compulsive Symptoms OBS - Obsessing ORDER - Ordering HOARD - Hoarding NEUTRALIZE - Neutralizing

Figure 1: Confirmatory factor analysis path diagram depicting the item loadings for the 6 factors of the OCI-CV among the Nigerian adolescents $(n=1017)$; Indices of fitness: $\mathrm{IFI}=0.917$; CFI $=0.916$; $\mathrm{NFI}=0.906 ;$ SRMR $=0.041$;MSEA $=0.086 / 90 \% \mathrm{CI}=0.082-$ 0.096

\section{Correlational analyses between OCI-CV and other study measures $(n=1017)$}

Table 3 showed that there were statistically significant positive correlations between OCI-CV and HADS-Anxiety $(\mathrm{rp}=0.75, \mathrm{p}<0.001)$ and HADS-Depression $(\mathrm{rp}=0.72$, $\mathrm{p}<0.001)$. However, there was a statistically significant negative correlation between OCI-CV and Self-Esteem (rp $=-0.72, \mathrm{p}<0.001)$. In addition, there was also a statistically significant modest positive correlation between OCI-CV and GHQ (rp $=0.57, \mathrm{p}<0.001)$ and Age ( $\mathrm{p} p=0.37, \mathrm{p}<0.001)$. However, not shown in table 3 , all the OCI-CV subscales had significant positive correlations with the HADS-Anxiety 
and Depression subscales, GHQ-12 with age, and significant negative correlations with self-esteem.

Table 3: Correlational analyses between OCI-CV total and other study measures $(n=1017)$

\begin{tabular}{llllll}
\hline Variables & 1 & 2 & 3 & 4 & 5 \\
& & & & & \\
\hline OCl-CV total & 1 & & & & \\
HADS-Anxiety & $0.75^{*}$ & 1 & & & \\
HADS-Depression & $0.72^{*}$ & $0.82^{*}$ & 1 & & \\
Self-Esteem & $-0.72^{*}$ & $-0.79^{*}$ & $-0.85^{*}$ & 1 & \\
GHQ & $0.57^{*}$ & $0.57^{*}$ & $0.58^{*}$ & $-0.61^{*}$ & 1 \\
Age & $0.37^{*}$ & $0.32^{*}$ & $0.34^{*}$ & $-0.32^{*}$ & $0.25^{\star}$ \\
\hline
\end{tabular}

$* p<0.001$

Mean OCl-CV total and subscale scores in relation to gender and adolescent age category (middle and late adolescence)

There were no differences in the mean OCI-CV total and subscale scores between the male and female adolescent respondents: Doubting / Checking - male $=1.33(\mathrm{SD} 2.23)$, female $=1.33(\mathrm{SD} 2.17), \mathrm{t}=-0.025, \mathrm{p}=-0.980$; Obsessing male $=1.04(\mathrm{SD} 1.79)$, female $=1.11(\mathrm{SD} 1.82), \mathrm{t}=-0.582$, $\mathrm{p}=0.561 ;$ Hoarding - male $=0.79(\mathrm{SD} 1.36)$, female $=0.72$ $(1.23), \mathrm{t}=0.975, \mathrm{p}=0.330$; Washing - male $=1.02(\mathrm{SD} 1.65)$, female $=1.06(1.68), \mathrm{t}=-0.362, \mathrm{p}=0.717$; Ordering - male $=0.84(\mathrm{SD} 1.61)$, female $=1.00(1.71), \mathrm{t}=-1.517, \mathrm{p}=0.130$; Neutralizing - male $=0.62($ SD 1.22), female $=0.66(1.19), \mathrm{t}$ $=-0.569, \mathrm{p}=0.570 ;$ OCI $-\mathrm{CV}$ total - male $=5.65(\mathrm{SD} 9.03)$, female $=5.88(8.82), \mathrm{t}=-0.409, \mathrm{p}=0.683$.

In relation to the adolescent age category, the respondents in the late adolescent category (17-19 years, $n=153(15.1 \%)$, had significantly higher mean OCI-CV total and subscale scores compared to those in the middle adolescent category (13-16 years, $\mathrm{n}=864$ (84.9\%): Doubting / Checking - middle adolescence $=1.17(\mathrm{SD} 2.11)$, late adolescence $=2.22(\mathrm{SD}$ 2.45), $\mathrm{t}=-5.484, \mathrm{p}<0.001 ;$ Obsessing - middle adolescence $=$ 0.97 (SD 1.74), late adolescence $=1.71(\mathrm{SD} 2.03), \mathrm{t}=-4.769$, $\mathrm{p}<0.001$; Hoarding - middle adolescence $=0.67$ (SD 1.23), late adolescence $=1.21(\mathrm{SD} 1.44), \mathrm{t}=-4.746, \mathrm{p}<0.001$; Washing - middle adolescence $=0.92(\mathrm{SD} 1.59)$, late adolescence $=1.73(\mathrm{SD} 1.88), \mathrm{t}=-5.660, \mathrm{p}<0.001 ;$ Ordering - middle adolescence $=0.81(\mathrm{SD} 1.58)$, late adolescence $=1.55(\mathrm{SD}$ 1.97), $\mathrm{t}=-5.098, \mathrm{p}<0.001$; Neutralizing - middle adolescence $=0.57(\mathrm{SD} 1.13)$, late adolescence $=1.05(\mathrm{SD} 1.49), \mathrm{t}=$ $-4.633, \mathrm{p}<0.001$; OCI-CV total - middle adolescence $=5.11$ $(\mathrm{SD}$ 8.56), late adolescence $=9.47(\mathrm{SD} 9.98), \mathrm{t}=-5.651$, $\mathrm{p}<0.001$.

Linear regression analysis showing the variables that predicted OCI-CV subscale scores among the respondents $(n=1017)$
Table 4: Linear regression (hierarchical regression) showing the variables that predicted OCI-CV subscale scores among the respondents $(n=1017)$

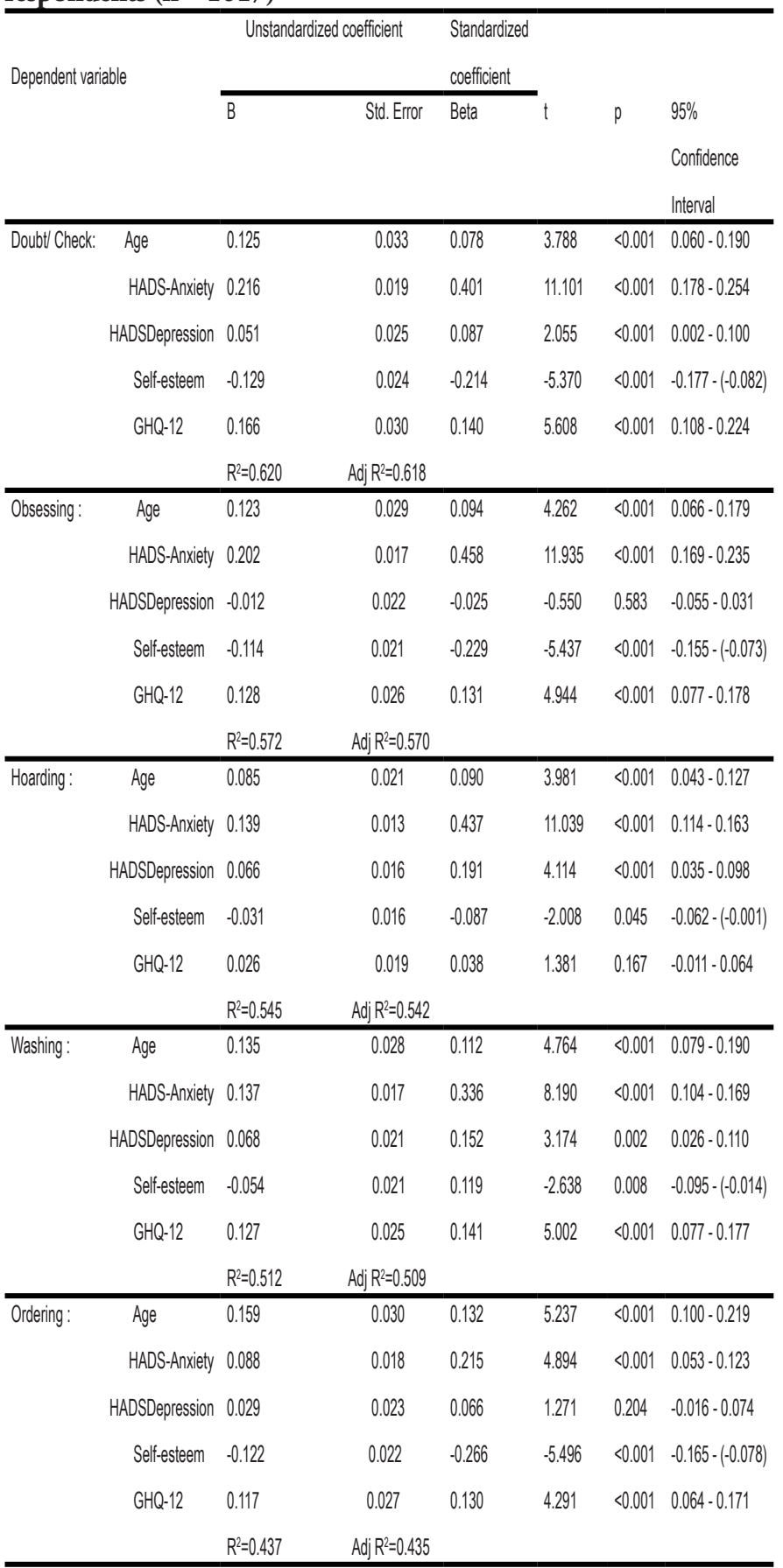

Table 4 shows the variables that significantly predicted the OCI-CV-subscale scores. It can be observed that for the Doubting / Checking subscale, a combination of age, anxiety and depressive symptoms, self-esteem and psychological distress accounted for approximately $62 \%$ of its variance; for the Obsessing subscale, age, anxiety symptoms, selfesteem and psychological distress cumulatively accounted for $57 \%$ of its variance. The combination of age, anxiety and depressive symptoms and self-esteem aggregately accounted for $54.2 \%$ of the variance in the Hoarding subscale. In the Washing subscale, approximately $51 \%$ of its variance was accounted for by the combination of age, anxiety and depressive symptoms, self-esteem and psychological distress. In the Ordering subscale, approximately $44 \%$ of its variance was accounted for by age, anxiety symptoms, self-esteem and psychological distress, while for the Neutralizing subscale, the combination of age, anxiety and depressive symptoms, self-esteem and psychological distress accounted for 
approximately $42 \%$ of its variance.

\section{Discussion}

This is the first study in Nigeria and sub-Saharan Africa to examine the psychometric properties of the OCI-CV in a cross-sectional sample of adolescents. The primary aims of this study were to confirm the factor structure, and examine the reliability and validity in addition to other correlates of the OCI-CV in a non-clinical sample of Nigerian adolescents. The overall internal consistency of the Inventory's 21 items in this study was 0.96. The internal consistency of the OCI-CV was examined by calculating the McDonald's Omega value rather than the Cronbach's alpha which has been described as a statistically flawed estimate of a scale's reliability ${ }^{44}$. This overall reliability value was higher than what the original developers of the Inventory reported ${ }^{26}$. A number of previous authors in developed countries have also reported comparable overall internal consistency of the OCI-CV among samples of adolescents ${ }^{28,33}$. The majority of the previous studies that examined the psychometric characteristics of the OCI-CV had internal consistency (Cronbach's alpha) values ranging from 0.71 to $0.94^{26,30,33}$.

The Hoarding (0.71) and Neutralizing (0.69) subscales of the OCI-CV had moderate internal consistencies which are still within the acceptable range in the context of a non-clinical sample ${ }^{45}$. In this study, the lower internal consistency values observed in relation to the Hoarding and Neutralizing subscales are similar to what other studies have reported $26,28,30,33$. However, a study that involved a cross-sectional sample of Spanish adolescents reported a much lower internal consistency (Cronbach's alpha) value of 0.43 for the Hoarding subscale ${ }^{28}$. We can, therefore, to a reasonable extent, conclude that the OCI-CV and its 6 subscales have demonstrated satisfactory reliabilities among Nigerian adolescents. Confirmatory factor analyses of the OCI-CV items demonstrated a model fit with indices that were rather poor compared to what was described by the original developers of the Inventory ${ }^{26}$. The poor indices of fitness we observed among the Nigerian adolescents were similar to what was reported in a study that examined the psychometric properties of the OCI-CV among a sample of American adolescents ${ }^{29}$. In our study, it was also noted among the Nigerian adolescents that despite the poor indices of fitness on CFA, each of the items that constituted the 6 subscales had satisfactory factor loading. Concerning age and gender differences, there has been some controversies. In this study, late adolescent (17-19 years) respondents had higher mean scores in all subscales compared to those in the middle adolescent (13-16 years) group. This possibly showed that OCS increases with age. This is consistent with studies with both clinical and community-based samples that showed higher scores among older children ${ }^{26,30,46}$. Furthermore, there were no gender differences in this study. This is also consistent with previous findings that reported no gender differences ${ }^{26,28,47}$. On the contrary, other studies reported gender differences ${ }^{30,46}$.

The concurrent validity of the OCI-CV in our study was supported by its significant correlations with the Anxiety and Depression subscales of the HADS, and the Rosenberg Self-Esteem Scale and the Psychological distress scale (GHQ-12). We noted that the directions and strengths of the correlations between the OCI-CV and these other measures were as we expected. One other observation that gives credence to the concurrent validity of the OCI$\mathrm{CV}$ was that on linear regression analyses, the Anxiety and Depression subscales of the HADS were the only study measures that significantly determined the OCI-CV scores among the Nigerian adolescents. Previous studies that examined the validity of the OCI-CV have reported similar significant correlations with other anxiety and depression measuring scales ${ }^{26,28,30}$. Thus, the OCI-CV has demonstrated satisfactory concurrent validity among our sample of Nigeria adolescents. There were no statistically significant differences between the male and female adolescents in terms of total OCI-CV score and subscale scores. This is similar to what has been reported by other studies ${ }^{26,28}$. However, a study in Chile reported that female adolescents had higher mean total OCI-CV scores ${ }^{30}$. The significant positive correlations we observed between age and total OCI-CV scores among the Nigerian adolescents in this study is supported by what was reported among Chilean adolescents, in which the authors indicated that older adolescents had higher total OCI-CV scores compared to the younger ones ${ }^{30}$. In this study, the prevalence of OCS among Nigerian adolescents was $37.8 \%$. In Egypt, various studies have reported prevalence rates of $13.3 \%, 18.4 \%$ and $43.1 \%$ with measures other than the OCI$\mathrm{CV}^{12-14}$. About a decade ago, a study in Nigeria reported that approximately $40 \%$ of Nigerian students may exhibit $\mathrm{OCS}^{48}$. The variations in the prevalence of OCS among adolescents in the few studies in Egypt and Nigeria could be due to methodological issues relating to the measures that were adopted.

\section{Limitations of the study}

This study is not without limitations. Caution must be exercised in generalizing the results of this study to other parts of the country or other African countries, since the study was conducted among adolescents in south-western region of Nigeria. However, the likelihood of differences in psychometric properties of the OCI-CV among adolescents with similar sociodemographic profiles in other parts of the country is not likely. Another limitation was that we adopted a non-clinical sample; hence, further studies involving clinical samples of Nigerian adolescents are still needed to examine the psychometric properties of the OCI-CV, especially in terms of its feasibility as a diagnostic instrument. Also, further studies are still needed to determine the Inventory's test-retest reliability and discriminant validity as well as the age and gender differences in other regions of the country. Despite these limitations, this study has a few strengths. First, we recruited a relatively large sample size of nonclinical adolescents. The sample size in our study exceeds that of some of the other previous studies that had assessed the psychometric properties of OCI-CV ${ }^{26,28,30}$.

\section{Conclusion}

The results of this study have evidenced that the OCI-CV possesses satisfactory reliability and validity among Nigerian adolescents, despite the modestly acceptable indices of fitness of the CFA model. We believe that this study will serve as an initial step at identifying OCS among the population of Nigerian adolescents through the availability of a psychometrically valid and reliable scale such as the OCI-CV. 


\section{Acknowledgements}

We appreciate the staff and students of the high schools where the research was conducted.

\section{Source of funding}

None

\section{Conflict of Interest}

None declared.

\section{References}

1. Ruscio A, Stein D, Chiu W, Kessler R. The epidemiology of obsessivecompulsive disorder in the National Comorbidity Survey Replication. Mol Psychiatry. 2010;15(1):53-63. doi: 10.1038/mp.2008.94.

2. Farrell L, Barrett P. Obsessive-compulsive disorder across developmental trajectory: Cognitive processing of threat in children, adolescents and adults. $\mathrm{Br} \mathrm{J}$ Psychol. 2006;97(1):95-114. doi: $10.1348 / 000712605 \times 58592$.

3. Geller DA, March J. Practice parameter for the assessment and treatment of children and adolescents with obsessive-compulsive disorder. J Am Acad Child Adolesc Psychiatry. 2012;51(1):98-113. doi: 10.1016/j.jaac.2011.09.019

4. Barrett PM, Healy LJ. An examination of the cognitive processes involved in childhood obsessive-compulsive disorder. Behav Res Ther. 2003;41(3):285-99.

5. Farrell L, Waters A, Milliner E, Ollendick T. Comorbidity and treatment response in pediatric obsessive-compulsive disorder: a pilot study of group cognitive-behavioral treatment. Psychiatry Res. 2012;199(2):115-23. doi: 10.1016/j.psychres.2012.04.035.

6. Alvarenga PG, Cesar RC, Leckman JF, Moriyama TS, Torres AR, Bloch $\mathrm{MH}$, et al. Obsessive-compulsive symptom dimensions in a population-based, cross-sectional sample of school-aged children. J Psychiatr Res. 2015;62:108-14. doi: 10.1016/j.jpsychires.2015.01.018.

7. Bryńska A, Wolańczyk T. Epidemiology and phenomenology of obsessive-compulsive disorder in non-referred young adolescents. Eur Child Adolesc Psychiatry. 2005;14(6):319-27. doi:10.1007/s00787005-0478-3.

8. Canals J, Hernández-Martínez C, Cosi S, Voltas N. The epidemiology of obsessive-compulsive disorder in Spanish school children. J Anxiety Disord. 2012;26(7):746-52. doi: 10.1016/j.janxdis.2012.06.003.

9. Shams G, Foroughi E, Esmaili Y, Amini H, Ebrahimkhani N. Prevalence rates of obsessive-compulsive symptoms and psychiatric comorbidity among adolescents in Iran. Acta Med Iran. 2011;49(10):680-7.

10. Sun J, Boschen MJ, Farrell LJ, Buys N, Li Z-J. Obsessivecompulsive symptoms in a normative Chinese sample of youth: Prevalence, symptom dimensions, and factor structure of the Leyton Obsessional Inventory - Child Version. J Affect Disord. 2014;164:1927. doi: 10.1016/j.jad.2014.04.004.

11. Valleni-Basile LA, Garrison CZ, Jackson KL, Waller JL, McKEOWN RE, Addy CL, et al. Frequency of obsessive-compulsive disorder in a community sample of young adolescents. J Am Acad of Child Adolesc Psychiatry. 1994;33(6):782-91. doi: 10.1097/00004583199407000-00002.

12. Humaida M, Ismail I, Abd-Alfattah M, Eisa A. Prevalence rates of obsessive-compulsive symptoms, obsessive-compulsive disorder, and psychiatric comorbidity among secondary-school students in rural Egypt. Int J Ment Health Addict (internet). 2004 Nov (cited 2018 Jan). Available from: http://www.ijma-journal.com/pdf/c01a19.pdf

13. Okasha A, Ragheb K, Attia A, Seif eDA, Okasha T, Ismail R. Prevalence of obsessive compulsive symptoms (OCS) in a sample of Egyptian adolescents. Encephale. 2000;27(1):8-14.

14. Rady A, Salama H, Wagdy M, Ketat A. Obsessive compulsive phenomenology in a sample of Egyptian adolescent population. Eur J Psychiatry. 2013;27(2):89-96. doi: 10.4321/S021361632013000200002

15. Voltas N, Hernández-Martínez C, Arija V, Aparicio E, Canals J. A Prospective Study of Paediatric Obsessive-Compulsive Symptomatology in a Spanish Community Sample. Child Psychiatry Hum Dev. 2014;45(4):377-87. doi:10.1007/s10578-013-0408-4.

16. Helbing M-LC, Ficca M. Obsessive-compulsive disorder in school-age children. J Sch Nurs. 2009;25(1):15-26. doi: $10.1177 / 1059840508328199$.

17. Alonso P, Segalas C, Real E, Pertusa A, Labad J, Jiménez-Murcia $\mathrm{S}$, et al. Suicide in patients treated for obsessive-compulsive disorder: A prospective follow-up study. J Affect Disord. 2010;124(3):300-8. doi: 10.1016/j.jad.2009.12.001

18. Balci V, Sevincok L. Suicidal ideation in patients with obsessivecompulsive disorder. Psychiatry Res. 2010;175(1):104-8. doi: 10.1016/j. psychres.2009.03.012.

19. Storch EA, Lewin AB, Larson MJ, Geffken GR, Murphy TK, Geller DA. Depression in youth with obsessive-compulsive disorder: clinical phenomenology and correlates. Psychiatry Res. 2012;196(1):83-9. doi:10.1016/j.psychres.2011.10.013.

20. Abramowitz JS, Storch EA, Keeley M, Cordell E. Obsessivecompulsive disorder with comorbid major depression: what is the role of cognitive factors? Behav Res Ther. 2007;45(10):2257-67. doi:10.1016/j.brat.2007.04.003.

21. Husain N, Chaudhry I, Ahmed G. Self-esteem and obsessive compulsive disorder. J Pak Med Assoc. 2014;64(1):64-8.

22. Jakubovski E, Diniz JB, Valerio C, Fossaluza V, Belotto-Silva $\mathrm{C}$, Gorenstein $\mathrm{C}$, et al. Clinical predictors of long-term outcome in obsessive-compulsive disorder. Depress Anxiety. 2013;30(8):763-72. doi: 10.1002/da.22013.

23. Scahill L, Riddle MA, McSwiggin-Hardin M, Ort SI, King RA, Goodman WK, et al. Children's Yale-Brown obsessive compulsive scale: reliability and validity. J Am Acad Child Adolesc Psychiatry. 1997;36(6):844-52. doi:10.1097/00004583-199706000-00023.

24. Storch EA, Bagner D, Merlo LJ, Shapira NA, Geffken GR, Murphy TK, et al. Florida obsessive-compulsive inventory: Development, reliability, and validity. J Clin Psychol. 2007;63(9):851-9. doi:10.1002/ jclp.20382.

25. Berg CJ, Rapoport JL, Flament M. The Leyton obsessional inventory-child version. J Am Acad Child Psychiatry. 1986;25(1):8491.

26. Foa EB, Coles M, Huppert JD, Pasupuleti RV, Franklin ME, March J. Development and validation of a child version of the obsessive compulsive inventory. Behavior Res Ther. 2010;41(1):121-32. doi:10.1016/j.beth.2009.02.001.

27. Bloch MH, Landeros-Weisenberger A, Rosario MC, Pittenger C, Leckman JF. Meta-analysis of the symptom structure of obsessivecompulsive disorder. Am J Psychiatry. 2008;165(12):1532-42. doi:10.1176/appi.ajp.2008.08020320.

28. Alcázar AIR, Ruiz García B, Iniesta-Sepúlveda M, Parada Navas JL, Rosa-Alcázar Á, Parada-Navas JL. Obsessive Compulsive InventoryChild Version (OCI-CV) in a Spanish community sample of children and adolescents. Psicothema. 2014;26(2):174-179. doi: 10.7334/ psicothema2013.210

29. Jones AM, De Nadai AS, Arnold EB, McGuire JF, Lewin AB, Murphy TK, et al. Psychometric properties of the obsessive compulsive inventory: child version in children and adolescents with obsessivecompulsive disorder. Child Psychiatry Hum Dev. 2013;44(1):137-51. doi: 10.1007/s10578-012-0315-0.

30. Martínez-González AE, Rodríguez-Jiménez T, Piqueras JA, VeraVillarroel P, Godoy A. Psychometric properties of the ObsessiveCompulsive Inventory-Child Version (OCI-CV) in Chilean children 
and adolescents. PloS ONE. 2015;10(8):e0136842. doi: 10.1371/ journal.pone.0136842.

31. Rodriguez-Jimenez T, Piqueras JA, Lázaro L, Moreno E, Ortiz AG, Godoy A. Metric invariance, reliability, and validity of the Child Version of the Obsessive Compulsive Inventory (OCI-CV) in community and clinical samples. J Obsessive Compuls Relat Disord. 2016;9:1-8 doi: 10.1016/j.jocrd.2016.01.003

32. Rodríguez-Jiménez T, Godoy A, Piqueras JA, Gavino A, MartínezGonzález AE, Foa EB. Factor structure and measurement invariance of the Obsessive-Compulsive Inventory-Child Version (OCI-CV) in general population. Eur J Psychol Assess. 2017;33(2):97-103. doi:10.1027/1015-5759/a000276.

33. Pozza A, Barcaccia B, Dèttore D. The Obsessive Compulsive Inventory-Child Version (OCI-CV): Further Evidence on Confirmatory Factor Analytic Structure, Incremental and Criterion Validity in Italian Community Children and Adolescents. Archives Psychiatr Nurs. 2017;31(3):291-5. doi:10.1016/j.apnu.2017.02.003.

34. Opakunle T, Akinsulore A, Aloba OO, Fatoye FO. Obsessivecompulsive symptoms in schizophrenia: prevalence and associated factors in a Nigerian population. Int $\mathrm{J}$ Psychiatry Clin Pract. 2017:21(3):195-200. doi: 10.1080/13651501.2017.1330417.

35. Zigmond AS, Snaith RP. The hospital anxiety and depression scale. Acta Psychiatr Scand. 1983;67(6):361-70.

36. Abiodun O. A validity study of the Hospital Anxiety and Depression Scale in general hospital units and a community sample in Nigeria. Br J Psychiatry. 1994;165(5):669-72.

37. Goldberg D. Manual of the general health questionnaire. Windsor, UK, Natural Foundation for Educational Research: Nelson, 1978.

38. Gureje O. Reliability and the factor structure of the Yoruba version of the 12-item General Health Questionnaire. Acta Psychiatr Scand. 1991;84(2):125-9.

39. Goldberg DP, Gater R, Sartorius N, Ustun TB, Piccinelli M, Gureje $\mathrm{O}$, et al. The validity of two versions of the GHQ in the WHO study of mental illness in general health care. Psychol Med. 1997;27(1):191-7.

40. Rosenberg M. Society and the adolescent self-image. 1st ed, New Jersey: Princeton University Press. 1965.
41. Oyefeso AO, Zacheaus A. Self-esteem as Determined by Gender Differences among Yoruba Adolescents to Ibadan, Nigeria: A Research Note. J Child Psychol Psychiatry. 1990;31(3):461-3.

42. Kline RB. Principles and practice of structural equation modeling. 4th ed. New York: Guilford publications; 2015.

43. Tabachnick B, Fidel I. LS. Using multivariate statistics. 5th ed. Boston, MA: Allyn \& Bacon/ Pearson Education, 2007.

44. Peters GJY. The alpha and the omega of scale reliability and validity: why and how to abandon Cronbach's alpha and the route towards more comprehensive assessment of scale quality. Eur Health Psychol. 2014;16(2):56-69.

45. Gliner JA, Morgan GA, Leech NL. Research methods in applied settings: An integrated approach to design and analysis. 2nd ed. New York: Routledge, 2011.

46. Rodríguez-Jiménez T, Godoy A, Piqueras JA, Gavino A, MartínezGonzález AE, Foa EB. Factor structure and measurement invariance of the Obsessive-Compulsive Inventory-Child version (OCI-CV) in general population. Eur J Psychol Assess. 2015;33(2):97-103. doi: 10.1027/1015-5759/a000276

47. Jones AM, De Nadai AS, Arnold EB, McGuire JF, Lewin AB, Murphy TK, et al. Psychometric properties of the obsessive compulsive inventory: child version in children and adolescents with obsessivecompulsive disorder. Child Psychiatry Hum Dev. 2013;44(1)137-151. doi: 10.1007/s10578-012-0315-0.

48. Uwakwe R. Obbessive-compulsive symptoms in a sample of Nigerian undergraduate students: comparison with two instruments. J Biomed Inv. 2006;4(1):1-8. doi: 10.4314/jbi.v4i1.30407 